\title{
Studies With Enamines: Synthesis and Reactivity of \\ 4-Nitrophenyl-1-piperidinostyrene. Synthesis of Pyridazine, Oxadiazole, 1,2,3-Triazole and 4-Aminopyrazole Derivatives
}

Tayseer A. Abdallah, Abdellatif M. Salaheldin, and Naglaa F. Radwan

Department of Chemistry, Faculty of Science, Cairo University, Giza, A. R. Egypt

Reprint requests to Dr. A. M. Salaheldin. E-mail: amsalaheldin@yahoo.com

Z. Naturforsch. 2007, 62b, 261 -266; received October 19, 2006

4-Nitrophenyl-1-piperidinostyrene (4) reacts with an aromatic diazonium salt to afford the arylhydrazonal 6. The latter condenses with active methylene compounds to yield pyridazine derivatives, and with hydroxylamine hydrochloride to produce oxadiazole and 1,2,3-triazole derivatives. Compound 12 was reacted with chloroacetonitrile to afford 4-aminopyrazoles $\mathbf{1 5}$.

Key words: 2-Arylhydrazononitriles, Pyridazinimine, Oxadiazole, 1,2,3-Triazole,

4-Aminopyrazoles

\section{Introduction}

Enamines are versatile reagents that are currently utilized extensively as synthetic equivalents of aldehydes $[1-5]$. In earlier work we have shown that functionally substituted enamines are versatile precursors to otherwise not readily obtainable active methylene aldehydes [6-8]. In conjunction with this work, we report here a synthesis of the title compound $\mathbf{4}$ and its use as a 4-nitrophenylacetaldehyde equivalent for the synthesis of different heteroaromatic compounds such as pyridazines, 4-aminopyrazoles and 1,2,3-triazoles (Scheme 1). The strategy adopted herein for the synthesis of the target compound $\mathbf{4}$ is based on the use of 4-nitrophenylacetic acid (2).

\section{Result and Discussion}

The reaction of 4-nitrophenylacetic acid (2) with triethyl orthoformate and piperidine in DMF solution afforded the enamine $\mathbf{4}$ in good yield. Utilization of triethyl orthoformate and piperidine in DMF solution was found more economic and safer than using DMFDMA. It is believed that piperidine reacts with acetal $\mathbf{1}$ which then condenses with $\mathbf{2}$ to yield $\mathbf{3}$, which is readily decarboxylated into the final isolated product $\mathbf{4}$ as depicted in Scheme 1.

The possibility that decarboxylation preceded condensation was ruled out based on the failure of attempts to condense 4-nitrotoluene with orthoformate under the same reaction conditions. Although it was earlier reported that nitrotoluenes condense with DMFDMA to yield enamines [9], we failed to repeat this work. The ${ }^{1} \mathrm{H}$ NMR spectrum of compound 4 showed two singlet signals for the piperidinyl protons at $\delta=1.64$ $\left(3 \mathrm{CH}_{2}\right)$ and $3.20\left(2 \mathrm{CH}_{2}\right)$ ppm and two doublets at $\delta=$ 5.30 and $6.96 \mathrm{ppm}$ for the two olefinic protons. The olefinic coupling constant $\left({ }^{3} \mathrm{~J}=14 \mathrm{~Hz}\right)$ indicated that the reaction product exists solely in the $E$-form 4 . The

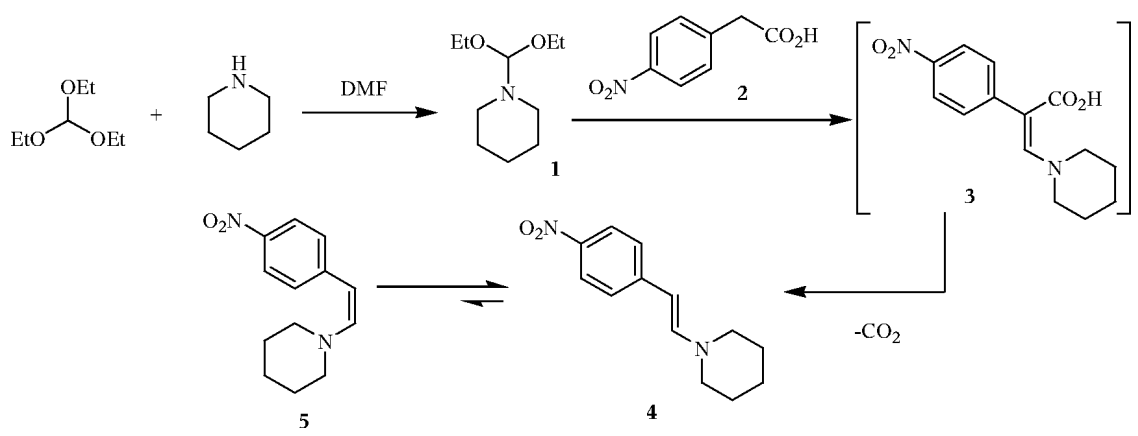

Scheme 1. 


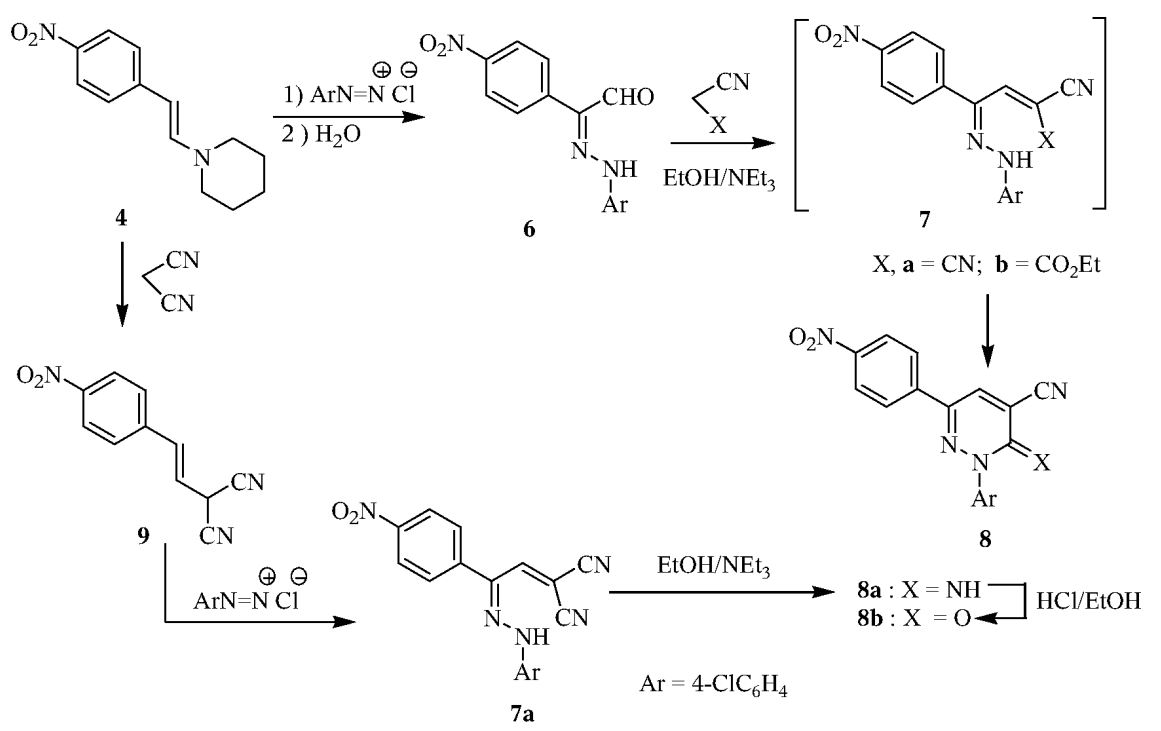

Scheme 2.

$Z$-isomer 5 was not detected by NMR. ${ }^{13} \mathrm{C}$ NMR and mass spectra of compound $\mathbf{4}$ are in accordance with the proposed structure.

The considerable biological activities of pyridazine derivatives have incited considerable interest in developing efficient synthetic approaches for differently substituted pyridazine derivatives [10-13]. Compound 4 proved to be a perfect synthetic equivalent for 4-nitrophenylacetaldehyde to produce pyridazine derivatives. Thus, it coupled readily with aromatic diazonium salts to yield the arylhydrazonals 6 . It reacted with malononitrile and ethyl cyanoacetate to afford compounds $\mathbf{8 a}, \mathbf{b}$. Establishing of their structures was based on the elemental analyses and spectral data. Compound 8a was converted quantitively into pyridazinone $\mathbf{8 b}$ by treatment with ethanolic hydrochloric acid solution as shown in Scheme $2[14,15]$.

Establishing of structure $\mathbf{8}$ was based on the spectral analysis. For example, the IR spectra of compound 8a showed an NH stretching band at $3363 \mathrm{~cm}^{-1}$ and a strong band at $2196 \mathrm{~cm}^{-1}$ for the $\mathrm{CN}$ group. In case of $\mathbf{8 b}$, a $\mathrm{C}=\mathrm{O}$ absorption band at $1681 \mathrm{~cm}^{-1}$ in addition to a $\mathrm{CN}$ band at $2235 \mathrm{~cm}^{-1}$ was found. The ${ }^{1} \mathrm{H}$ NMR spectra of compound $\mathbf{8 a}$ revealed, in addition to the aromatic protons, the presence of a singlet at $\delta=$ $7.63 \mathrm{ppm}$ corresponding to pyridazinimine $4-\mathrm{H}$ and a singlet at $\delta=8.58 \mathrm{ppm}$ corresponding to NH. The mass spectra of the products are in accordance with the proposed structure ( $c f$. Experimental Section).

The structures of compounds $\mathbf{8 a}, \mathbf{b}$ were confirmed by their alternative synthesis via refluxing compound 4 with malononitrile in ethanol in the presence of a catalytic amount of triethylamine to yield compound 9 which was coupled with an aromatic diazonium salt to afford compound 7a. Compound 7a was cyclized into the pyridazinimine 8a by heating in ethanolic triethylamine solution (m. p., mixed m. p. and TLC control). Compound $8 \mathbf{a}$ could be converted into $\mathbf{8 b}$ by refluxing compound $\mathbf{8 a}$ in ethanolic hydrochloric acid as indicated in Scheme $2[14,15]$.

The utility of 2-arylhydrazonoaldehydes in heterocyclic synthesis has received a considerable interest in the past decade $[7,8]$. Compound 6 reacted with hydroxylamine hydrochloride in ethanol in the presence of triethylamine to afford the oxime 10, similar to an earlier report on arylhydrazonals [7]. In refluxing aqueous acetic acid and in the presence of sodium acetate, compound 6 reacted with hydroxylamine hydrochloride to yield 2-arylhydrazononitrile $\mathbf{1 2}$, formed via intermediacy of the oxime acetate $\mathbf{1 1}$.

Compound $\mathbf{1 2}$ has also been obtained from treatment of oxime $\mathbf{1 1}$ with acetic acid/ammonium acetate solution. It is believed that $\mathbf{1 1}$ was acylated in acetic acid solution and that the acylated compound $\mathbf{1 1}$ derivative underwent thermal elimination of acetic acid to yield 12 (Scheme 3).

Treatment of compound $\mathbf{6}$ with acetic anhydride at reflux afforded the oxadiazole derivative $\mathbf{1 3}$, whereas refluxing of $\mathbf{1 0}$ in acetic anhydride yielded the 1,2,3triazole derivative 14. The structures of 13 and 14 were established based on their elemental analysis and spectral data. For example, the ${ }^{1} \mathrm{H}$ NMR spectrum of com- 


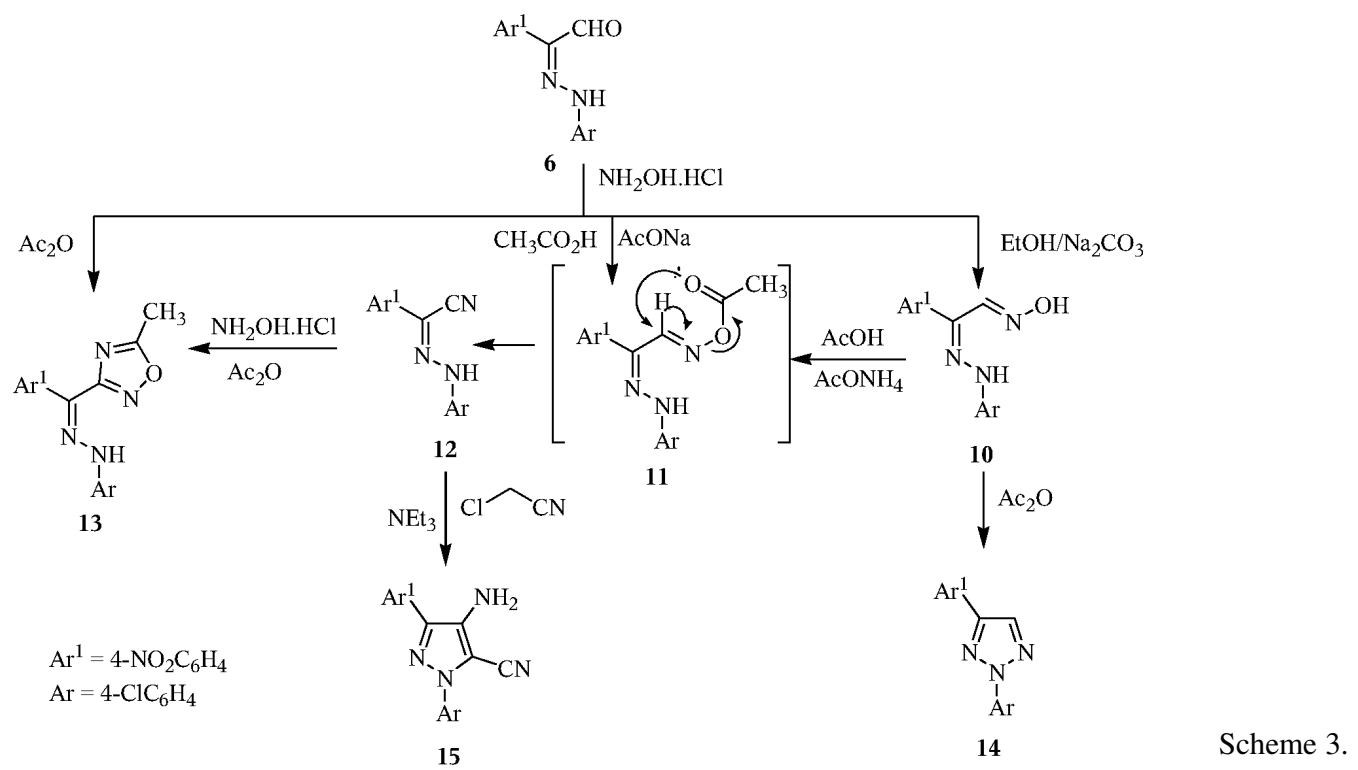

pound 13 revealed the presence of a singlet at $\delta=$ $2.66 \mathrm{ppm}$ corresponding to a $\mathrm{CH}_{3}$ group, a multiplet at $\delta=7.30-8.35 \mathrm{ppm}$ corresponding to aromatic protons and a singlet at $\delta=9.86 \mathrm{ppm}$ corresponding to NH. The mass spectrum of the product was consistent with these results. Again compound 13 could also be obtained by refluxing the 2-aryl-hydrazononitrile 12 with hydroxylamine hydrochloride in acetic anhydride for $4 \mathrm{~h}$ (m.p., mixed m.p. and TLC control). The ${ }^{1} \mathrm{H}$ NMR spectrum of compound 14 revealed in addition to the aromatic protons a singlet at $\delta=8.15 \mathrm{ppm}$ corresponding to the triazole proton $5-\mathrm{H}$.

Compound 12, so formed, proved to be a valuable precursor to arylazoles. For example, reacting 12 with chloroacetonitrile afforded 4-aminopyrazole $\mathbf{1 5}$, relatives of which have been described recently [16].

\section{Experimental Section}

All melting points were measured with a Gallenkamp electrothermal melting point apparatus and are uncorrected. IR spectra were recorded as $\mathrm{KBr}$ pellets with a Pye Unicam SP 3-300 Spectrophotometer. ${ }^{1} \mathrm{H}$ and ${ }^{13} \mathrm{C}$ NMR spectra were recorded in deuterated dimethylsulfoxide $\left[\mathrm{D}_{6}\right] \mathrm{DMSO}$ or deuterated chloroform $\left(\mathrm{CDCl}_{3}\right)$ at $300 \mathrm{MHz}$ with a Varian Gemini NMR spectrometer using tetramethylsilane (TMS) as an internal reference, and results are expressed as $\delta$ values. Mass spectra were performed on a Shimadzu GCMS-QP $1000 \mathrm{Ex}$ mass spectrometer at $70 \mathrm{eV}$. Elemental analyses were carried out at the Microanalytical Center of Cairo University.

\section{4-Nitrophenyl-1-piperidinostyrene (4)}

A mixture of 4-nitrophenyl acetic acid $(0.5 \mathrm{~mol})$, triethyl orthoformate $(0.5 \mathrm{~mol})$ and piperidine $(0.5 \mathrm{~mol})$ was treated with $50 \mathrm{~mL}$ of DMF and refluxed for $72 \mathrm{~h}$. The reaction mixture was then cooled to r.t. and poured onto water. The solid product, so formed, was collected by filtration and crystallized from ethanol. M. p. $98-99{ }^{\circ} \mathrm{C}$. Yield: $75 \%$. - IR (KBr): $v=2853$ (CH aliphatic), $1629(\mathrm{C}=\mathrm{C}) \mathrm{cm}^{-1} .-{ }^{1} \mathrm{H} \mathrm{NMR}$ (300 MHz, $\left.\mathrm{CDCl}_{3}, 25^{\circ} \mathrm{C}, \mathrm{TMS}\right): \delta=1.64\left(\mathrm{~s}, 6 \mathrm{H}, 3 \mathrm{CH}_{2}\right.$ ), $3.20\left(\mathrm{~s}, 4 \mathrm{H}, 2 \mathrm{CH}_{2}\right), 5.30(\mathrm{~d}, J=14 \mathrm{~Hz}, 1 \mathrm{H}, \mathrm{CH}), 6.94(\mathrm{~d}$, $J=14 \mathrm{~Hz}, 1 \mathrm{H}, \mathrm{CH}), 7.16(\mathrm{~d}, J=9 \mathrm{~Hz}, 2 \mathrm{H}, \mathrm{Ar}-\mathrm{H}), 8.04(\mathrm{~d}$, $J=9 \mathrm{~Hz}, 2 \mathrm{H}, \mathrm{Ar}-\mathrm{H}) .-{ }^{13} \mathrm{C}$ NMR $\left(75 \mathrm{MHz},\left[\mathrm{D}_{6}\right] \mathrm{DMSO}\right)$ : $\delta=23.8,25.2,48.9,94.7(\mathrm{HC}=\mathrm{CH}), 122.2,124.2,141.1$ $(\mathrm{HC}=\mathrm{CH}), 144.7,149.0 .-\mathrm{MS}(\mathrm{EI}, 70 \mathrm{eV}): m / z(\%)=232$ (36) $\left[\mathrm{M}^{+}\right.$]. $-\mathrm{C}_{13} \mathrm{H}_{16} \mathrm{~N}_{2} \mathrm{O}_{2}$ (232.27): calcd. C 67.22, H 6.94, N 12.06; found C 66.95, H 6.80, N 12.22.

\section{2-[2-(4-Chlorophenyl)hydrazono]-2-(4-nitrophenyl)- acetaldehyde (6)}

A cold solution of arenediazonium chloride $(10 \mathrm{mmol})$ was prepared by adding a solution of sodium nitrite $(10 \mathrm{mmol}$ in $2 \mathrm{~mL}$ of $\mathrm{H}_{2} \mathrm{O}$ ) to a cold solution of the aromatic amine hydrochloride with stirring. The resulting solution of the arenediazonium chloride was added to a cold solution of 1(4-nitrostyryl)piperidine (4) in ethanol $(50 \mathrm{~mL})$ containing sodium acetate $(5 \mathrm{~g})$. The reaction mixture was stirred at r.t. for $30 \mathrm{~min}$. The solid product, so formed, was collected by filtration, washed with water and crystallized from ethanol. M. p. $210-212{ }^{\circ} \mathrm{C}$. Yield: $70 \%$. - IR (KBr): $v=3423(\mathrm{NH})$, $1700(\mathrm{C}=\mathrm{O}) \mathrm{cm}^{-1} .-{ }^{1} \mathrm{H}$ NMR $\left(300 \mathrm{MHz},\left[\mathrm{D}_{6}\right] \mathrm{DMSO}\right.$, $25{ }^{\circ} \mathrm{C}$, TMS $): \delta=7.16(\mathrm{~d}, J=9 \mathrm{~Hz}, 2 \mathrm{H}, \mathrm{Ar}-\mathrm{H}), 7.41(\mathrm{~d}$, 
$J=9 \mathrm{~Hz}, 2 \mathrm{H}, \mathrm{Ar}-\mathrm{H}), 7.66(\mathrm{~d}, J=9 \mathrm{~Hz}, 2 \mathrm{H}, \mathrm{Ar}-\mathrm{H}), 8.32(\mathrm{~d}$, $J=9 \mathrm{~Hz}, 2 \mathrm{H}, \mathrm{Ar}-\mathrm{H}), 9.56$ (s, 1H, CHO), $11.0(\mathrm{~s}, 1 \mathrm{H}, \mathrm{NH})$. MS (EI, $70 \mathrm{eV}): m / z(\%)=303(72)\left[\mathrm{M}^{+}\right], 305(23)\left[\mathrm{M}^{+}+2\right]$. $-\mathrm{C}_{14} \mathrm{H}_{10} \mathrm{ClN}_{3} \mathrm{O}_{3}$ (303.7): calcd. C 55.37, $\mathrm{H}$ 3.32, N 13.84, Cl 11.67; found C 55.26, H 3.10, N 13.75, Cl 11.60.

\section{2-[2-[(4-Chlorophenyl)hydrazono]-2-(4-nitrophenyl) ethylidene]malononitrile ( $7 a)$}

A cold solution of arenediazonium chloride $(10 \mathrm{mmol})$ was prepared by adding a solution of sodium nitrite $(10 \mathrm{mmol}$ in $2 \mathrm{~mL}$ of $\mathrm{H}_{2} \mathrm{O}$ ) to a cold solution of the aromatic amine hydrochloride with stirring. The resulting solution of the arenediazonium chloride was added to a cold solution of $\mathbf{9}$ in ethanol $(50 \mathrm{~mL})$ containing sodium acetate $(5 \mathrm{~g})$. The reaction mixture was stirred at $\mathrm{r}$.t. for $30 \mathrm{~min}$. The solid product, so formed, was collected by filtration, washed with water and crystallized from ethanol. M.p. $130-132{ }^{\circ} \mathrm{C}$. Yield: $72 \%$. - IR (KBr): $v=3340(\mathrm{NH}), 2220,2208(\mathrm{CN})$, $1610(\mathrm{C}=\mathrm{C}) \mathrm{cm}^{-1}$. - MS (EI, $\left.70 \mathrm{eV}\right): \mathrm{m} / \mathrm{z}(\%)=351(36)$ $\left[\mathrm{M}^{+}\right], 353$ (11) $\left[\mathrm{M}^{+}+2\right] .-\mathrm{C}_{17} \mathrm{H}_{10} \mathrm{ClN}_{5} \mathrm{O}_{2}$ (351.74): calcd. C 58.05, H 2.87, N 19.91, Cl 10.08; found C 57.95, H 3.05, N 19.77, Cl 10.18.

General procedure for the preparation of pyridazine derivatives $8 a, b$

Method A: a mixture of 2-arylhydrazonal $6(10 \mathrm{mmol})$ and malononitrile or ethyl cyanoacetate (10 mmol of each) was refluxed in ethanol $(50 \mathrm{~mL})$ for $3 \mathrm{~h}$ in the presence of triethylamine. The solvent was evaporated in vacuo and the solid residue was collected by filtration and crystallized from ethanol.

Method B: To a solution of $7 \mathbf{a}(10 \mathrm{mmol})$ in $30 \mathrm{~mL}$ of ethanol was added $1 \mathrm{~mL}$ of triethylamine, and the solution was refluxed for $1 \mathrm{~h}$. After cooling to r. t. the reaction mixture was diluted with cold water and neutralized with hydrochloric acid. The precipitate was collected by filtration and crystallized to afford a product, which was identical to $\mathbf{8 a}$ in all respects.

\section{Transformation of $8 \boldsymbol{a}$ to $8 \boldsymbol{b}$}

To a solution of $3.03 \mathrm{~g}(10 \mathrm{mmol})$ of $\mathbf{8 a}$ in $30 \mathrm{~mL}$ of ethanol was added conc. $\mathrm{HCl}(5 \mathrm{~mL})$ and the solution was refluxed for $1 \mathrm{~h}$. After cooling to $\mathrm{r}$. t. the reaction mixture was diluted with cold water and neutralized with ammonia. The precipitate was collected by filtration and crystallized to afford a product, which was identical to $\mathbf{8 b}$ in all respects.

\section{2-(4-Chlorophenyl)-2,3-dihydro-3-imino-6-(4-nitro- phenyl)pyridazine-4-carbonitrile $(8 a)$}

M.p. $175-177{ }^{\circ} \mathrm{C}$. Yield: $70 \%$. - IR (KBr): $v=3363$ (NH), $2196(\mathrm{CN}) \mathrm{cm}^{-1} .-{ }^{1} \mathrm{H}$ NMR $\left(300 \mathrm{MHz},\left[\mathrm{D}_{6}\right] \mathrm{DMSO}\right.$, $\left.25{ }^{\circ} \mathrm{C}, \mathrm{TMS}\right): \delta=7.20-7.53(\mathrm{~m}, 4 \mathrm{H}, \mathrm{Ar}-\mathrm{H}), 7.63$ (s, $1 \mathrm{H}$,
5-H), $7.85-8.27(\mathrm{~m}, 4 \mathrm{H}, \mathrm{Ar}-\mathrm{H}), 8.58(\mathrm{~s}, 1 \mathrm{H}, \mathrm{NH}) .-\mathrm{MS}$ $($ EI, $70 \mathrm{eV}): m / z(\%)=351(100)\left[\mathrm{M}^{+}\right], 353(33)\left[\mathrm{M}^{+}+2\right]$. $-\mathrm{C}_{17} \mathrm{H}_{10} \mathrm{ClN}_{5} \mathrm{O}_{2}$ (351.7): calcd. C 58.05, H 2.87, N 19.91, $\mathrm{Cl} 10.08$; found $\mathrm{C} 57.80, \mathrm{H} 2.80, \mathrm{~N} 20.17, \mathrm{Cl} 10.30$.

\section{2-(4-Chlorophenyl)-2,3-dihydro-6-(4-nitrophenyl)-3-oxo- pyridazine-4-carbonitrile $(8 \mathrm{~b})$}

M.p. $270-272{ }^{\circ} \mathrm{C}(\mathrm{EtOH} / \mathrm{DMF}, 3: 1)$. Yield: $74 \%$. IR $(\mathrm{KBr}): v=2235(\mathrm{CN}), 1681(\mathrm{C}=\mathrm{O}) \mathrm{cm}^{-1} .-{ }^{1} \mathrm{H}$ NMR (300 MHz, [D 6 ]DMSO, $25{ }^{\circ} \mathrm{C}$, TMS): $\delta=7.56(\mathrm{~d}, J=9 \mathrm{~Hz}$, 2H, Ar-H), 7.92 (d, $J=9 \mathrm{~Hz}, 2 \mathrm{H}, \mathrm{Ar}-\mathrm{H}), 8.19$ (d, $J=9 \mathrm{~Hz}$, 2H, Ar-H), 8.35 (d, J=9 Hz, 2H, Ar-H), 9.08 (s, 1H, 5-H). MS (EI, $70 \mathrm{eV}): m / z(\%)=352(75)\left[\mathrm{M}^{+}\right], 354(23)\left[\mathrm{M}^{+}+2\right]$. $-\mathrm{C}_{14} \mathrm{H}_{10} \mathrm{ClN}_{3} \mathrm{O}_{3}$ (352.73): calcd. C 57.89, H 2.57, N 15.88, Cl 10.05; found C 57.66, H 2.70, N 15.75, Cl 10.30.

\section{2-[2-(4-Nitrophenyl)vinyl]malononitrile (9)}

A mixture of 4 (2.32 g, $10 \mathrm{mmol})$, malononitrile (0.66 g, $10 \mathrm{mmol})$ and triethylamine $(1.01 \mathrm{~g}, 10 \mathrm{mmol})$ in ethanol $(25 \mathrm{~mL})$ was refluxed for $3 \mathrm{~h}$. The solvent was evaporated under vacuum, and the crude product was collected and crystallized from ethanol. M. p. $80-81{ }^{\circ} \mathrm{C}$. Yield: $80 \%$. - IR (KBr): $v=2201(\mathrm{CN}), 1601(\mathrm{C}=\mathrm{C}) \mathrm{cm}^{-1} \cdot-{ }^{1} \mathrm{H}$ NMR $(300 \mathrm{MHz}$, [D $\mathrm{D}_{6}$ DMSO, $\left.25{ }^{\circ} \mathrm{C}, \mathrm{TMS}\right): \delta=4.44(\mathrm{~s}, 1 \mathrm{H}, \mathrm{CH}), 7.25(\mathrm{~d}$, $J=9 \mathrm{~Hz}, 1 \mathrm{H}, \mathrm{CH}), 7.80(\mathrm{~d}, J=9 \mathrm{~Hz}, 1 \mathrm{H}, \mathrm{CH}), 7.90-8.35$ $(\mathrm{m}, 4 \mathrm{H}, \mathrm{Ar}-\mathrm{H}) .-\mathrm{MS}(\mathrm{EI}, 70 \mathrm{eV}): m / z(\%)=213(31)\left[\mathrm{M}^{+}\right]$. $-\mathrm{C}_{11} \mathrm{H}_{7} \mathrm{~N}_{3} \mathrm{O}_{2}$ (213.19): calcd. C 61.97, H 3.31, N 19.71; found C 61.65, H 3.35, N 19.75.

\section{2-(4-Chlorophenylhydrazono)-2-(4-nitrophenyl)ethanal-1- oxime (10)}

A warm solution of hydroxylamine hydrochloride $(0.69 \mathrm{~g}$, $10 \mathrm{mmol})$ and sodium carbonate $(1.26 \mathrm{~g}, 12 \mathrm{mmol})$ in water $(10 \mathrm{~mL})$ was added to a stirred solution of the arylhydrazonoethanal $(6)(10 \mathrm{mmol})$ in ethanol $(4 \mathrm{~mL})$. The reaction mixture was stirred at r.t. for $4 \mathrm{~h}$. The oximes soon separated as semisolid crystals that were solidified by cooling in crushed ice. The solid product, so formed, was collected by filtration and crystallized from ethanol/DMF $(3: 1)$. M. p. $270-272{ }^{\circ} \mathrm{C}$. Yield: $77 \%$. - IR (KBr): $v=3365(\mathrm{OH})$, $3206(\mathrm{NH}) \mathrm{cm}^{-1} .-{ }^{1} \mathrm{H}$ NMR $\left(300 \mathrm{MHz},\left[\mathrm{D}_{6}\right] \mathrm{DMSO}, 25{ }^{\circ} \mathrm{C}\right.$, TMS): $\delta=7.05(\mathrm{~d}, J=9 \mathrm{~Hz}, 2 \mathrm{H}, \mathrm{Ar}-\mathrm{H}), 7.20(\mathrm{~s}, 1 \mathrm{H}, \mathrm{CH})$, 7.44 (d, $J=9 \mathrm{~Hz}, 2 \mathrm{H}, \mathrm{Ar}-\mathrm{H}), 7.60$ (d, $J=10 \mathrm{~Hz}, 2 \mathrm{H}, \mathrm{Ar}-\mathrm{H})$, 8.16 (d, $J=10 \mathrm{~Hz}, 2 \mathrm{H}, \mathrm{Ar}-\mathrm{H}), 8.24$ (s, 1H, NH), 8.88 (s, 1H, OH). - MS (EI, $70 \mathrm{eV}): m / z(\%)=318(80)\left[\mathrm{M}^{+}\right], 320(26)$ $\left[\mathrm{M}^{+}+2\right] .-\mathrm{C}_{14} \mathrm{H}_{11} \mathrm{ClN}_{4} \mathrm{O}_{3}$ (318.75): calcd. C 52.76, H 3.48, $\mathrm{N}$ 17.58, Cl 11.12; found C 52.89, H 3.35, N 17.75, Cl 11.22.

(4-Chlorophenyl)hydrazono-(4-nitrophenyl)acetonitrile (12)

Method A: A mixture of hydroxylamine hydrochloride $(0.69 \mathrm{~g}, 10 \mathrm{mmol})$, sodium acetate $(3 \mathrm{~g})$ and arylhydrazo- 
noethanal $(6)(10 \mathrm{mmol})$ was refluxed in acetic acid $(20 \mathrm{~mL})$ for $4 \mathrm{~h}$. The solvent was removed under vacuum and the residue was poured onto water. The solid product was collected by filtration and crystallized from ethanol.

Method B: The oxime $\mathbf{1 0}(10 \mathrm{mmol})$ was refluxed in acetic acid $(10 \mathrm{~mL})$ and ammonium acetate $(3 \mathrm{~g})$ for $4 \mathrm{~h}$, and then left to cool at r.t. The solvent was reduced under vacuum and the residue was poured onto water. The solid product was collected by filtration and crystallized from ethanol. M. p. $200-201^{\circ} \mathrm{C}$. Yield: $80 \%$. - IR (KBr): $v=3380(\mathrm{NH})$, $2208(\mathrm{CN}) \mathrm{cm}^{-1} .-{ }^{1} \mathrm{H} \mathrm{NMR}\left(300 \mathrm{MHz},\left[\mathrm{D}_{6}\right] \mathrm{DMSO}, 25^{\circ} \mathrm{C}\right.$, TMS): $\delta=6.99-7.25(\mathrm{~m}, 4 \mathrm{H}, \mathrm{Ar}-\mathrm{H}), 7.30(\mathrm{~s}, 1 \mathrm{H}, \mathrm{NH}), 7.75$ (d, $J=10 \mathrm{~Hz}, 2 \mathrm{H}, \mathrm{Ar}-\mathrm{H}), 8.22$ (d, $J=10 \mathrm{~Hz}, 2 \mathrm{H}, \mathrm{Ar}-\mathrm{H})$. ${ }^{13} \mathrm{C}$ NMR (75 MHz, [D 6 DMSO): $\delta=157.2(C=\mathrm{N}), 155.2$, $144.1,137.4,130.2,129.1,124.5,123.4,120.2,117.2(C \mathrm{~N})$. - MS (EI, $70 \mathrm{eV}): m / z(\%)=300(25)\left[\mathrm{M}^{+}\right], 302(8)\left[\mathrm{M}^{+}+2\right]$. $-\mathrm{C}_{14} \mathrm{H}_{9} \mathrm{ClN}_{4} \mathrm{O}_{2}$ (300.70): calcd. C 55.92, H 3.02, N 18.63, Cl 11.79; found C 55.75, H 2.90, N 18.90, Cl 11.94.

\section{2-(4-Chlorophenyl)-1-(5-methyl-1,2,4-oxadiazol-3-yl)- (4-nitrophenyl)methylene hydrazine (13)}

Method A: A mixture of hydroxylamine hydrochloride $(0.69 \mathrm{~g}, 10 \mathrm{mmol})$, and arylhydrazonoethanal $(\mathbf{6})(10 \mathrm{mmol})$ was refluxed in acetic anhydride $(20 \mathrm{~mL})$ for $6 \mathrm{~h}$. The solvent was removed under vacuum and the residue was poured onto water. The solid product was collected by filtration and crystallized from ethanol.

Method B: A mixture of hydroxylamine hydrochloride $(0.69 \mathrm{~g}, 10 \mathrm{mmol})$ and arylhydrazononitrile $12(10 \mathrm{mmol})$ was refluxed in acetic anhydride $(10 \mathrm{~mL})$ for $4 \mathrm{~h}$, and then left to cool at r.t. The solvent was removed under vacuum and the residue was poured onto water. The solid product was collected by filtration and crystallized from ethanol.

M. p. $120-122{ }^{\circ} \mathrm{C}$. Yield: $55 \%$. - IR $(\mathrm{KBr}): v=3396$ (NH) $\mathrm{cm}^{-1} .-{ }^{1} \mathrm{H}$ NMR $\left(300 \mathrm{MHz}, \mathrm{CDCl}_{3}, 25{ }^{\circ} \mathrm{C}, \mathrm{TMS}\right)$ : $\delta=2.66\left(\mathrm{~s}, 3 \mathrm{H}, \mathrm{CH}_{3}\right), 7.30-7.60(\mathrm{~m}, 4 \mathrm{H}, \mathrm{Ar}-\mathrm{H}), 7.69-8.35$ (m, 4H, Ar-H), $9.86(\mathrm{~s}, 1 \mathrm{H}, \mathrm{NH}) .-\mathrm{MS}(\mathrm{EI}, 70 \mathrm{eV}): \mathrm{m} / z(\%)=$ 357 (13) $\left[\mathrm{M}^{+}\right.$]. $-\mathrm{C}_{16} \mathrm{H}_{12} \mathrm{ClN}_{5} \mathrm{O}_{3}$ (357.75): calcd. C 53.72, H 3.38, N 19.58, Cl 9.91; found C 53.60, H 3.40, N 19.38, $\mathrm{Cl} 9.60$.

\section{2-(4-Chlorophenyl)-4-(4-nitrophenyl)-2H-[1,2,3]triazole (14)}

The oxime $\mathbf{1 1}(10 \mathrm{mmol})$ was refluxed in acetic anhydride $(10 \mathrm{~mL})$ for $4 \mathrm{~h}$, and then left to cool at r.t. The solid product separated was collected by filtration and crystallized from ethanol. M.p. $150-152{ }^{\circ} \mathrm{C}$. Yield: $55 \%$. - IR (KBr): $v=$ 2925 (CH-Ar), $1601(\mathrm{C}=\mathrm{N}) \mathrm{cm}^{-1} .-{ }^{1} \mathrm{H}$ NMR $(300 \mathrm{MHz}$, $\left.\mathrm{CDCl}_{3}, 25^{\circ} \mathrm{C}, \mathrm{TMS}\right): \delta=7.48(\mathrm{~d}, J=9 \mathrm{~Hz}, 2 \mathrm{H}, \mathrm{Ar}-\mathrm{H}), 7.61$ (d, $J=9 \mathrm{~Hz}, 2 \mathrm{H}, \mathrm{Ar}-\mathrm{H}), 8.04(\mathrm{~d}, J=9 \mathrm{~Hz}, 2 \mathrm{H}, \mathrm{Ar}-\mathrm{H}), 8.32$ $(\mathrm{d}, J=9 \mathrm{~Hz}, 2 \mathrm{H}, \mathrm{Ar}-\mathrm{H}), 8.15(\mathrm{~s}, 1 \mathrm{H}$, triazole 5-H). - MS $(\mathrm{EI}, 70 \mathrm{eV}): \mathrm{m} / \mathrm{z}(\%)=300(69)\left[\mathrm{M}^{+}\right], 302(24)\left[\mathrm{M}^{+}+2\right]$. $-\mathrm{C}_{14} \mathrm{H}_{9} \mathrm{ClN}_{4} \mathrm{O}_{2}$ (300.75): calcd. C 55.92, H 3.02, N 18.63, Cl 11.79; found C 55.70, H 3.17, N 18.51, Cl 11.95.

\section{4-Amino-2-(4-chlorophenyl)-5-(4-nitrophenyl)-2H- pyrazole-3-carbonitrile (15)}

To a solution of $12(3.0 \mathrm{~g}, 10 \mathrm{mmol})$ in dioxane $(25 \mathrm{~mL})$ and triethylamine $(1.01 \mathrm{~g}, 10 \mathrm{mmol})$, chloroacetonitrile $(1 \mathrm{~mL}, 0.016 \mathrm{~mol})$ was added. The reaction mixture was refluxed for $3 \mathrm{~h}$ and then the solvent evaporated in vacuo; the solid product was filtered off and crystallized from ethanol. M.p. $187-188^{\circ} \mathrm{C}$. Yield: $85 \%$. - IR (KBr): $v=3451,3348$ $\left(\mathrm{NH}_{2}\right), 2217(\mathrm{CN}) \mathrm{cm}^{-1} .-{ }^{1} \mathrm{H} \mathrm{NMR}\left(300 \mathrm{MHz},\left[\mathrm{D}_{6}\right] \mathrm{DMSO}\right.$, $25{ }^{\circ} \mathrm{C}$, TMS): $\delta=6.49$ (s, $\left.2 \mathrm{H}, \mathrm{NH}_{2}\right), 7.25-7.48(\mathrm{~m}, 4 \mathrm{H}$, Ar-H), 7.55 (d, $J=10 \mathrm{~Hz}, 2 \mathrm{H}, \mathrm{Ar}-\mathrm{H}), 8.20(\mathrm{~d}, J=10 \mathrm{~Hz}$, $2 \mathrm{H}, \mathrm{Ar}-\mathrm{H}) .-{ }^{13} \mathrm{C}$ NMR (75 MHz, [D 6 DMSO): $\delta=145.2$, 143.0 (C-3), 141.6, 139.3, 137.6, 135.9 (C-4), 134.6, 128.8, 124.5, 122.2, $119.7(\mathrm{C}-5), 117.9(\mathrm{CN}) .-\mathrm{MS}(\mathrm{EI}, 70 \mathrm{eV}): \mathrm{m} / \mathrm{z}$ $(\%)=339(37)\left[\mathrm{M}^{+}\right], 341(11)\left[\mathrm{M}^{+}+2\right] .-\mathrm{C}_{16} \mathrm{H}_{10} \mathrm{ClN}_{5} \mathrm{O}_{2}$ (339.74): calcd. C 56.56, H 2.97, N 20.61, Cl 10.44; found C 56.42, H 3.10, N 20.30, Cl 10.50.
[1] B. Stanovinik, J. Svete, Chem. Rev. 2004, 104, 2433 2480.

[2] A. A. Aelaaser, A. A. El-Khair, Tetrahedron 2003, 59, $8463-8480$.

[3] K. M. Al-Zaydi, E. A. Hafez, M. H. Elnagdi, J. Chem. Res. (S), 2000, $154-155$, (M) 2000, 510-527.

[4] M. M. Abdel-Khalik, S.M. Agamy, M.H. Elnagdi, Synthesis 2001, $1861-1865$.

[5] N. A. Al-Awadi, M. H. Elnagdi, Y. A. Ibrahim, K. Kaul, A. Kumar, Tetrahedron 2001, 57, 1609-1614.

[6] M. A. Al-Shiekh, A. M. Salaheldin, E. A. Hafez, M. H. Elnagdi, J. Chem. Res. 2004, 3, 174-179.

[7] M. A. Al-Shiekh, A. M. Salaheldin, E. A. Hafez, M. H. Elnagdi, J. Heterocyclic Chem. 2004, 41, $647-$ 654.
[8] S. Almazroa, A. M. Salaheldin, M. H. Elnagdi, J. Heterocyclic Chem. 2004, 41, 267-272.

[9] M. G. Vetdino, J. W. Coe, Tetrahedron Lett. 1994, 35, $219-222$

[10] G. Negric, C. Kascheres, A. J. Kascheres, J. Heterocyclic Chem. 2004, 41, 461-491.

[11] O. O. Alekseeva, A. Mahadevan, J.L. Wiely, B.R. Martin, R. K. Razdan, Tetrahedron Lett. 2005, 46, $2159-2161$.

[12] R. F. Abdulla, R. S. Brinkmeyer, Tetrahedron 1979, 35, $1675-1735$.

[13] W. G. Coates, Pyridazines and their benzo derivatives, in Comprehensive Heterocyclic Chemistry. II, Vol. 6 (Eds.: A. R. Katrizky, K. W. Ress, F. V. Scriven) Pergamon, London, 1996, p. 87. 
[14] F. M. Abdelrazek, A. M. Salaheldin, A.E. Makky, Tetrahedron 2001, 57, 1813-1817.

[15] A. M. Salaheldin, Heteroatom Chem. 2003, 14, $612-$ 616.
[16] A. M. Salaheldin, T. A. Abdallah, N.F. Radwan, H. M. Hassaneen, Z. Naturforsch. 2006, 61b, $1158-$ 1161. 